\title{
Quantification of Composition of Epitaxial Si/SiGe by EELS
}

Michael A. Gribelyuk ${ }^{1}$, Wayne $\mathrm{Zhao}^{2}$ and Bianzhu $\mathrm{Fu}^{2,3}$

1. ATD Characterization, GLOBALFOUNDRIES, Malta, NY.

2. CCA Characterization, GLOBALFOUNDRIES, Malta, NY.

3. Currently at: Entegris Inc., Danbury, CT

Composition of epitaxial $\mathrm{Si} / \mathrm{SiGe}$ has been characterized in planar semiconductor devices by EDX with $1 \mathrm{~nm}$ resolution. Its accuracy was correlated to Secondary ion mass spectrometry and Auger electron spectroscopy to within 1 at.\% [1]. However, EDX analysis of SiGe in 3-D semiconductor devices is imprecise for the following reasons. First, physical thickness of epitaxial $\mathrm{Si} / \mathrm{SiGe}$ structures in $\mathrm{x}$ sectional TEM samples is less than 30nm. Since electron damage of SiGe limits the dose [1] EDX analysis of thin samples leads to variability in the quantified data. Second, some of the region behind SiGe film within $\mathrm{x}$-sectional TEM sample is filled with Si oxide. In addition, possible surface oxidation of the TEM sample plays a larger role in the EDX acquired signal. EDX analysis yields total Si and Ge content in the TEM sample (Fig. 1). Imprecise oxygen content as determined by EDX makes it difficult to subtract Si portion associated with the oxide from total Si content to determine Si fraction in the SiGe.

Since EELS offers superior sensitivity to light elements we explore quantification of SiGe composition by EELS. Both EELS and EDX data were acquired with a 200kV TEM instrument from the same TEM sample and quantified in terms of relative composition. EDX data was used to calibrate cross-sections of inelastic scattering $\sigma_{\mathrm{Si}}$ and $\sigma_{\mathrm{Ge}}$, which are used in EELS quantification. Planar Si/SiGe structure was used. Its thickness was sufficient to yield precise EDX composition, yet thin enough to avoid plural scattering effects in EELS measurement. Fig. 2 shows the Darkfield STEM image (a), EDX map (b) and relative composition across SiGe film (c). Relative composition was determined by EELS using Gatan Digital Micrograph GMS 2.1 EELS quantification software (Fig.3). It has yielded discrepancy as high as $7 \%$ as compared to EDX (i.e. $\mathrm{N}_{\mathrm{Ge}}=42 \%$ by EDX vs $\mathrm{N}_{\mathrm{Ge}}=49 \%$ by EELS). Cross-sections of inelastic scattering $\sigma_{\mathrm{Si}}=118$ barn and $\sigma_{\mathrm{Ge}}=327$ barn for Si K and Ge L2 edges computed by Digital Micrograph were used. The convergence semi-angle $\alpha=28.4 \mathrm{mrad}$ and collection semi-angle $\beta=88.4 \mathrm{mrad}$ were measured independently prior to the experiment. The integration window $\Delta=60 \mathrm{ev}$ was used for both edges for quantification. In the absence of plural scattering the composition ratio

$$
\frac{N_{G e}}{N_{S i}}=\frac{I_{G e}(\beta, \Delta) \sigma_{S i}}{I_{S i}(\beta, \Delta) \sigma_{G e}}
$$

The composition ratio $\mathrm{N}_{\mathrm{Ge}} / \mathrm{N}_{\mathrm{Si}}=0.74$ as per EDX. In contrast, $\mathrm{N}_{\mathrm{Ge}} / \mathrm{N}_{\mathrm{Si}}=0.96$ if cross-sections from Gatan Digital Micrograph were used. The TEM sample thickness $\mathrm{t}=60 \mathrm{~nm}$ as measured by EELS [2]. We found that removal of plural scattering in the acquired Spectrum Images using Fourier Log approach had no effect on the relative composition. The ratio of cross-sections of $\mathrm{Si}$ and $\mathrm{Ge} r=\sigma_{\mathrm{Si} /} \sigma_{\mathrm{Ge}}$ was increased by 1.3 to calibrate relative composition by EDX data. The relative composition of SiGe using corrected cross-sections $\sigma_{\mathrm{Si}}=118$ barn, $\sigma_{\mathrm{Ge}}=424$ barn is shown in Fig. 3e These values were applied for quantitative analysis of epitaxial $\mathrm{Si} / \mathrm{SiGe}$ films in structures with restricted geometry to be discussed in the presentation [3]. 
References:

[1] B. Fu et al, Microsc. Microanal. 22 (S3) (2016), p.1618.

[2] D.R.G. Mitchell, www.dmscripting.com, v2.1 Sept 2012, Mean Free Path Estimator, plugin for Digital Micrograph Software.

[3] The authors acknowledge Michael Hollner, Mark Hudson for preparation of TEM samples.
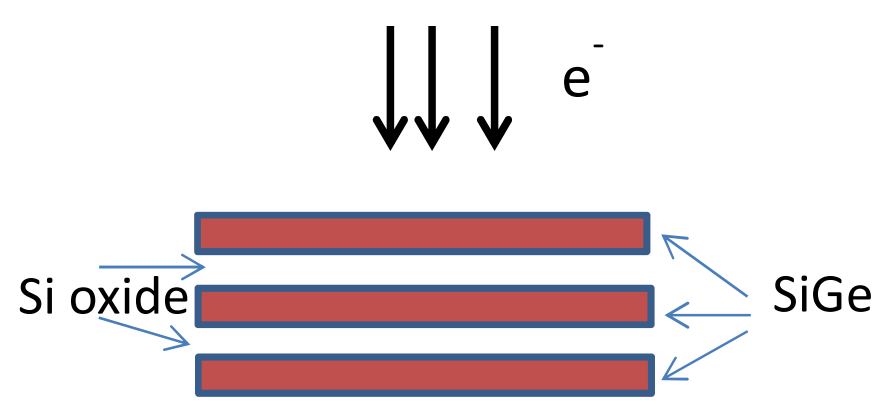

a

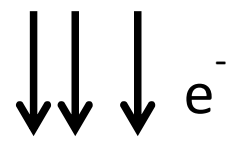

b

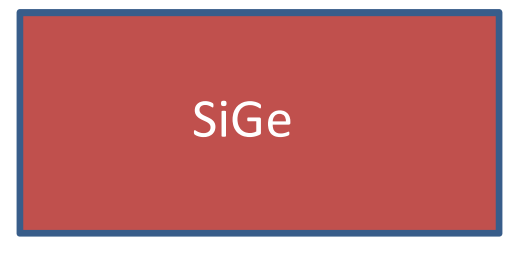

Figure 1. (a): Thin SiGe films are separated by Si oxide within TEM sample in 3-D devices.; (b): SiGe film is contnuous in the electron beam direction in planar structures
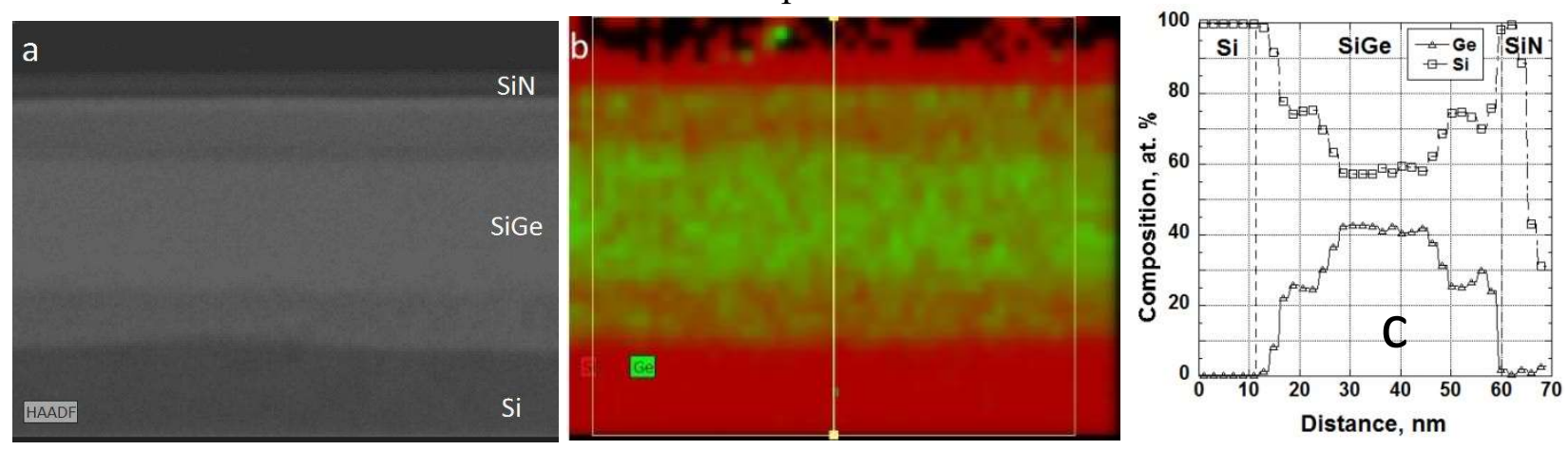

Figure 2. (a): STEM image, (b): EDX elemental map, (c): SiGe relative elemental composition in at.\% . Origin is at Si substrate.
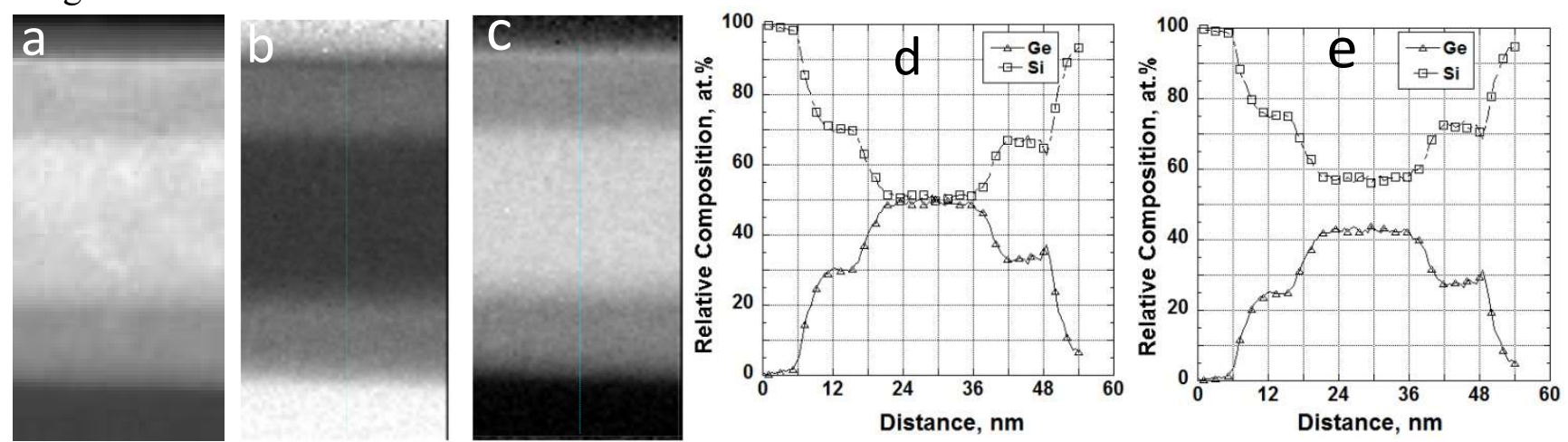

Figure 3. (a): STEM image; (b), (c): EELS relative composition of Si and Ge; (d): Relative elemental composition in at\%. Line in $(b, c)$ indicates location of line scan. Origin is at Si substrate. (e): Corrected relative elemental composition in at $\%$ 